\title{
Properties of Broad and Narrow Line Seyfert galaxies selected from SDSS
}

\author{
Vivek Kumar Jha*1, Hum Chand ${ }^{2}$, and Vineet Ojha ${ }^{1}$ \\ ${ }^{1}$ Aryabhatta research institute of observational sciences (ARIES), Nainital, Uttarakhand, India \\ ${ }^{2}$ Central University of Himachal Pradesh (CUHP), Dharamshala, Himachal Pradesh, India
}

\begin{abstract}
A comparative study of a representative sample of Broad and Narrow line Seyfert galaxies is presented. These galaxies have been selected from the $16^{\text {th }}$ data release of the Sloan Digital Sky Survey (SDSS-DR16). Some of the properties derived from single epoch spectrum vary significantly between the two populations. We find that the emission regions of Narrow line Seyfert galaxies are rich in iron content and the accretion rate is higher compared to the Broad line Seyfert galaxies. In our analysis, the $H \beta$ emission line is found to be asymmetric in few of the galaxies with more number of Narrow-line Seyfert 1 (NlSy1) galaxies showing blue asymmetries i.e. traces of outflowing gas as compared to the Broad-line Seyfert 1 (BlSy1) galaxies. This behaviour may be explained by the higher iron content present in the emission line regions of NlSy1 galaxies.
\end{abstract}

Keywords: galaxies: active - galaxies: nuclei-galaxies: Seyfert

\section{Introduction}

Seyfert galaxies are characterized by a lower luminous active nucleus as compared to the general quasar population. Among the Seyfert galaxies, Type-1 galaxies show both narrow and broad emission lines (see Netzer, 2015, for a review). These lower luminous Type-1 galaxies are subdivided into narrow and broad line Seyfert classes based on the Full Width at Half Maximum (FWHM) of the $H \beta$ emission line. NlSy1 galaxies are understood to be a subclass of active galactic nuclei (AGN) which have narrower broad Balmer line widths with FWHM of broad $H \beta$ emission line $\leq 2000 \mathrm{~km} / \mathrm{sec}^{-1}$, a small intensity ratio of the $[\mathrm{O}$ III $] \lambda 5007$ to $H \beta$ line ( $[O I I I] / H \beta \leq 3)$, stronger optical Fe II emissions (see Rakshit et al., 2017, and references therein), and usually steeper soft X-ray spectra and more rapid X-ray and sometimes optical flux variability (see Ojha et al., 2020a) . It is assumed that these properties are due to the central black hole being less massive, but accreting at a very high rate. Low optical variability has also been reported in NlSy1 galaxies by Rakshit \& Stalin (2017). It has been proposed by Mathur (2000) that the NISy1 galaxies are a subcategory of BlSy1 galaxies only and can be assumed to be in evolutionary stages, while in Gaskell (2000) unusually high Fe-II strength, $R_{f e}$ in NlSy1 galaxies is attributed to weak Balmer lines originating from a dense environment. The NlSy1 galaxies have been proposed as younger versions of the general broad line active galaxies.

The region responsible for the generation of broad emission lines is known as the Broad line Region (BLR). About 120 AGN been studied using the reverberation mapping technique (see Bahcall et al., 1972, Blandford \& McKee, 1982), which is a powerful time domain method to probe the inner regions of type 1 galaxies. However, with the known number of AGN extending into hundreds of thousands, thanks to the all sky surveys such as the Sloan Digital Sky Survey (SDSS) (York et al., 2000) but precise knowledge of the structure and kinematics of BLR through reverberation mapping available for only a handful of AGN (see Bentz \& Katz, 2015, for a comprehensive database of reverberation mapped AGN), studies have relied on statistical analysis on a selected sample of AGN constrained by

\footnotetext{
*vivek@aries.res.in, Corresponding author
} 


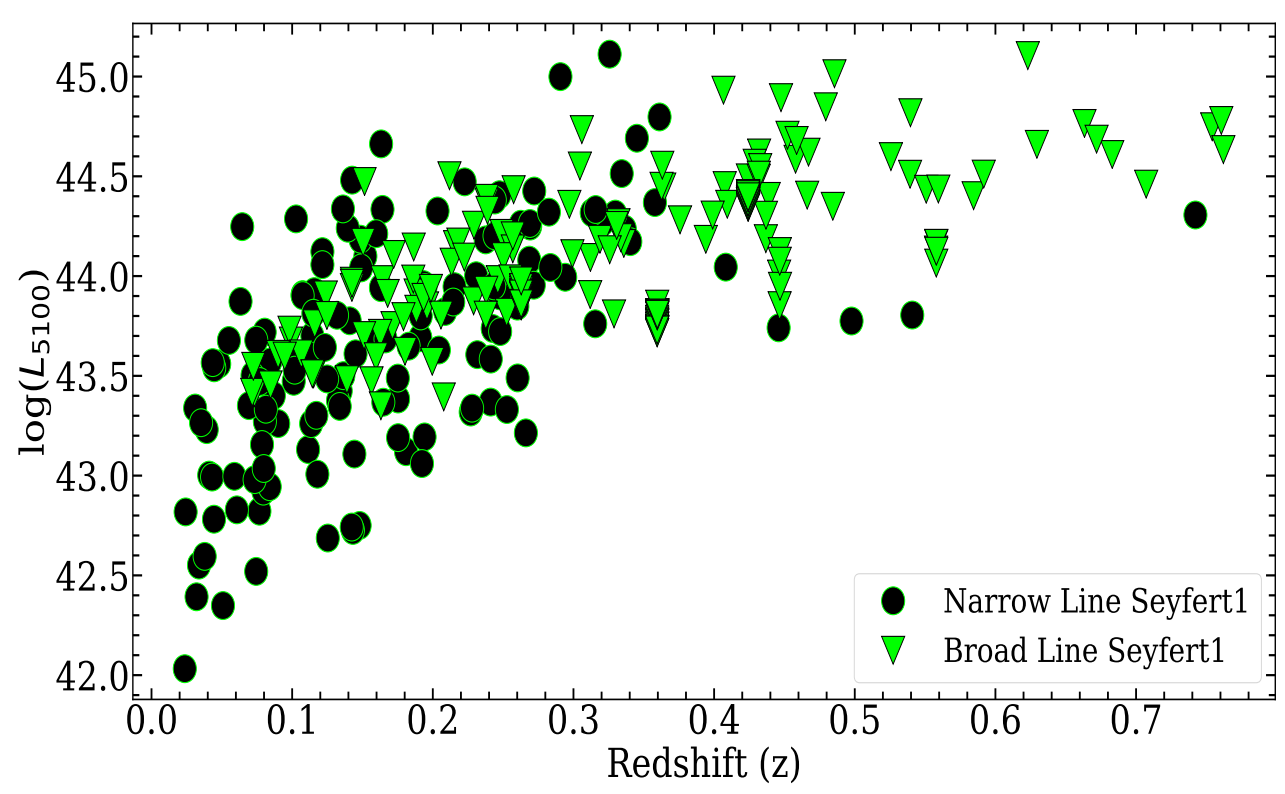

Figure 1. A representative sample of BlSy1 (green triangle) and the NlSy1 galaxies (black circle) galaxies matching in luminosity and redshift (L-z) plane being used for this work. The limit of redshift is put at 0.8 for clear $H \beta$ emission line detection in SDSS.

various limits, to infer the physical properties. A remarkable work was done by by Boroson \& Green (1992), where they performed a Principal Component Analysis (PCA) on properties derived from X-ray, optical and radio wavelength data for a set of 87 quasars. They derived that the Eigenvector1 (E1), driven by the anti correlation of the ratio $R_{F e}$ of equivalent width (EW) of FeII emission lines in the optical band and the FWHM of $H \beta$ emission line is the primary cause of variability in the parameters. Since then, various aspects of E1 involving a variety of data sets as well multi-frequency parameters have been discussed in multiple works (see Marziani et al., 2018, and references therein). Sulentic et al. (2000) and later Zamfir et al. (2010) established the foundations of the four-dimensional EV1 (4DE1) formalism, including the (FWHM) of $H \beta$ and $R_{f e}$ as two of the main components. These two quantities are respectively related to the black hole mass and the Eddington ratio and these studies have resulted in the so called quasar main sequence (see Marziani et al., 2018, Sulentic \& Marziani, 2015)

In this work, we have compiled a representative sample of NlSy1 galaxies and BlSy1 galaxies and performed a statistical study based on the various physical parameters responsible for driving the variations in both types of galaxies. The primary objective of current study is to understand the diversity observed in the physical parameters for a representative sample of both broad line and narrow line Seyfert-1 galaxies and its correlation with the physical parameters obtained through optical and X-ray observations, and then establish a comparison between the two types of galaxies based on these parameters.

\section{Data and fitting procedure}

A sample comprising of both BlSy1 and NlSy1 galaxies with almost similar luminosity and matching in the redshift domain has been assembled in order to study the properties of these two seemingly different classes of galaxies. More information about this sample is available in Ojha et al. (2020b). The single epoch optical spectrum for all the sources was obtained from the SDSS archives (see Rakshit et al., 2017, Shen et al., 2011, York et al., 2000, etc.) using the SDSS-SAS DR16 server ${ }^{1}$. For all the sources we performed a search query for the optical spectrum on the SDSS-SAS server for a region around 0.05 arc minutes within the specified Right Ascension (RA) and Declination (DEC) positions of these sources. The spectra were brought to the rest frame using the redshift values available in the

\footnotetext{
${ }^{1}$ https://dr16.sdss.org/optical/spectrum/search
} 


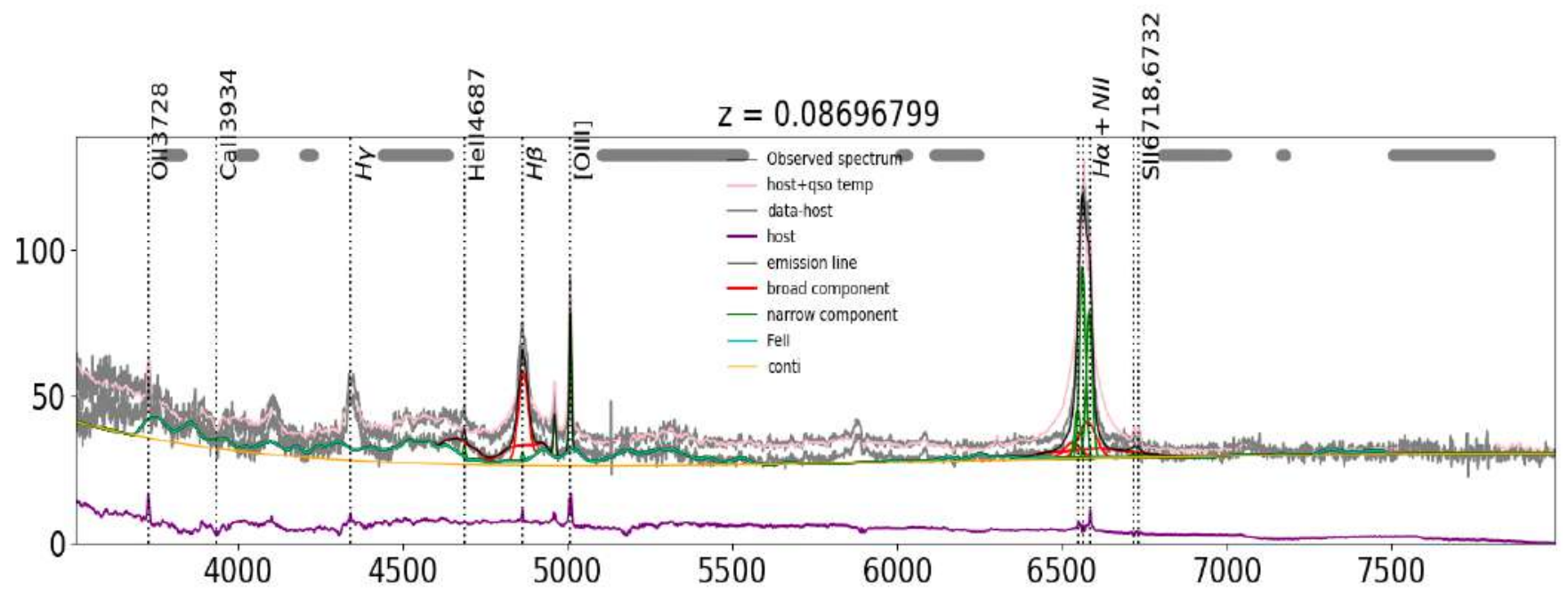

Figure 2. Demonstration of the fitting procedure. The continuum has been fit using a power law, the emission lines have been fit using a combination of gaussians, the host galaxy has been decomposed using already available tempelate in Yip et al. (2004) and the Fe blend has been removed using the tempelates available in Boroson \& Green (1992).

header of the individual FITS files obtained from SDSS DR16. A limit of Signal to Noise Ratio (SNR) $\geq 5$ was put for identifying the emission lines clearly. Out of the 225 NlSy1 galaxies in the sample, the SNR criteria reduced 10 sources while out of 164 BlSy1 galaxies from the sample, this criterion reduced 14 sources.

PyQSOFIT $^{2}$, a publicly available code to fit the quasar spectra has been used to analyse the individual spectra. It is written in Python language, and has been used for fitting the SDSS quasar spectra recently (see Gaur et al., 2019, Rakshit et al., 2020, etc.). Initially, the continuum model is prepared using the host galaxy components, contribution from the iron line and the accretion disk emission which reflects itself as a power law component (see Figure 2). We removed the host galaxy component using the PCA method, obtained from the host galaxy templates of (Yip et al., 2004) .For many of the sources in our sample the host galaxy decomposition could not be applied. The FeII blends were removed using the templates available in Boroson \& Green (1992) which are available within the code itself. The accretion disk component was fitted as a polynomial and the final continuum model was subtracted from the original spectrum, which yielded the emission line components only. We were concerned with measuring the asymmetry in the $H \beta$ emission line, hence for the emission line fitting, we concentrated on the $H \beta$-OIII complex only. We assumed the emission line complex to be composed of a narrow and a broad central component representing the $H \beta$ emission arising from the Narrow line and Broad line regions respectively. The width of the narrow Gaussian components used for fitting the OIII doublet, was tied with the narrow component of the $H \beta$ emission line which physically indicates the emission coming from the same narrow line region. The limits for the width of the Gaussian profiles were set as $800 \mathrm{~km} / \mathrm{sec}$ for narrow components, up to $2300 \mathrm{~km} / \mathrm{sec}$ for broad components and beyond $10000 \mathrm{~km} / \mathrm{sec}$ for very broad components for fitting the NlSy1 galaxies. The limit of 2300 $\mathrm{km} / \mathrm{sec}$ was kept keeping in mind the previous works classifying the NlSy1 galaxies (for example, see Rakshit et al., 2017). For fitting the BlSy1 we removed the upper limit of $2300 \mathrm{~km} / \mathrm{sec}$ on the broad component, while still allowing up to three Gaussian profiles including a very broad component. Out of $225 \mathrm{NlSy} 1$ galaxies, we could fit and get proper measurements of physical quantities for 144 sources, while out of 164 BlSy1 galaxies, we could fit and get proper measurements for 110 sources.

\footnotetext{
${ }^{2}$ https://github.com/legolason/PyQSOFit
} 


\section{Analysis}

We obtained the physical parameters from the spectral fitting and derived a few parameters based on empirical relations. The FWHM of the $H \beta$ emission line, area covered by the line, and its equivalent width (EW) were obtained from the direct decomposition of the spectra. The area covered by the $H \beta$ emission line was calculated by integrating the flux between $4700 \AA$ and $4920 \AA$. We calculated the equivalent width (EW) of the emission line using the same wavelength window and the monochromatic luminosity at $5100 \AA\left(L_{5100}\right)$ was obtained from the fit. The broad line region of NlSy1 galaxies is understood to be richer in FeII content as compared to the general population (Panda et al., 2019). The iron strength $\left(R_{F e}\right)$ was calculated as the ratio of area covered by the broad Fe line between $4433 \AA$ and $4684 \AA$, and the area covered by the $H \beta$ emission line.
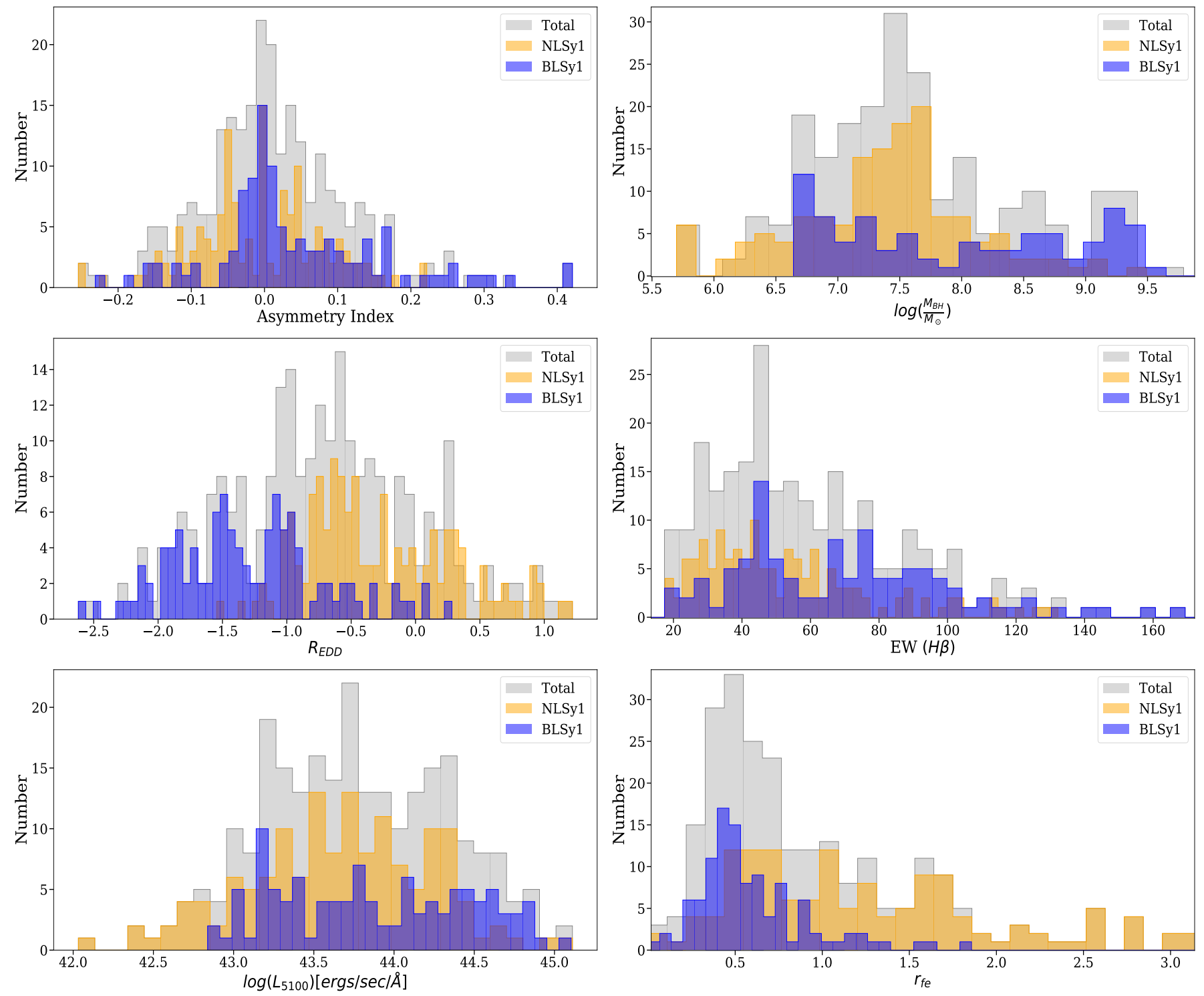

Figure 3. Distribution of various physical parameters for both the types of galaxies. Orange color denotes the NlSy1 galaxies, Blue color denotes the BlSy1 and the grey region is the combined number.

We found out the iron strength in NlSy1 galaxies to be higher than the BlSy1 galaxies for most of the AGN. The median $R_{F e}$ was 1.13 for the NlSy1 galaxies while it was less than half of that value, 0.49 for the BlSy1 galaxies. We estimated the flux ratio of the narrow OIII component to the broad $H \beta$ in order to understand the influence of the $H \beta$ on the emission from NLR gases. The NlSy1 galaxies have weak OIII emission and thus the OIII/H $\beta$ ratio was lower compared to the BlSy1 galaxies. Also, the ratio of broad components of $H \alpha$ and $H \beta$ was calculated using the area covered by the broad components of the two emission lines. 
The central black hole mass has been estimated using various empirical relations in the recent past. Reverberation mapping based masses provide tighter constraints on the Supermassive Black Hole (SMBH) mass and thus far this technique has been the only reliable one for SMBH mass estimation to higher redshifts (Bentz et al., 2009). The single epoch SMBH mass estimation technique is based on the scaling relations obtained from local galaxy stellar velocity dispersion.

$$
M_{B H}=f_{B L R} \frac{R_{B L R}(\Delta v)^{2}}{G}
$$

In this equation, $R_{B L R}$ is the BLR radius in light days and is estimated using the so called Radius Luminosity (R-L) relation available for a set of approximately 120 reverberation mapped AGN so far (Bentz et al., 2009, Du \& Wang, 2019), $\Delta v$ is obtained from the FWHM of the emission line being used for the SMBH mass estimation, assuming that the gas is in virialized motion around the SMBH. The NlSy1 galaxies have smaller SMBH mass, owing to the small FWHM of the $H \beta$ emission line.
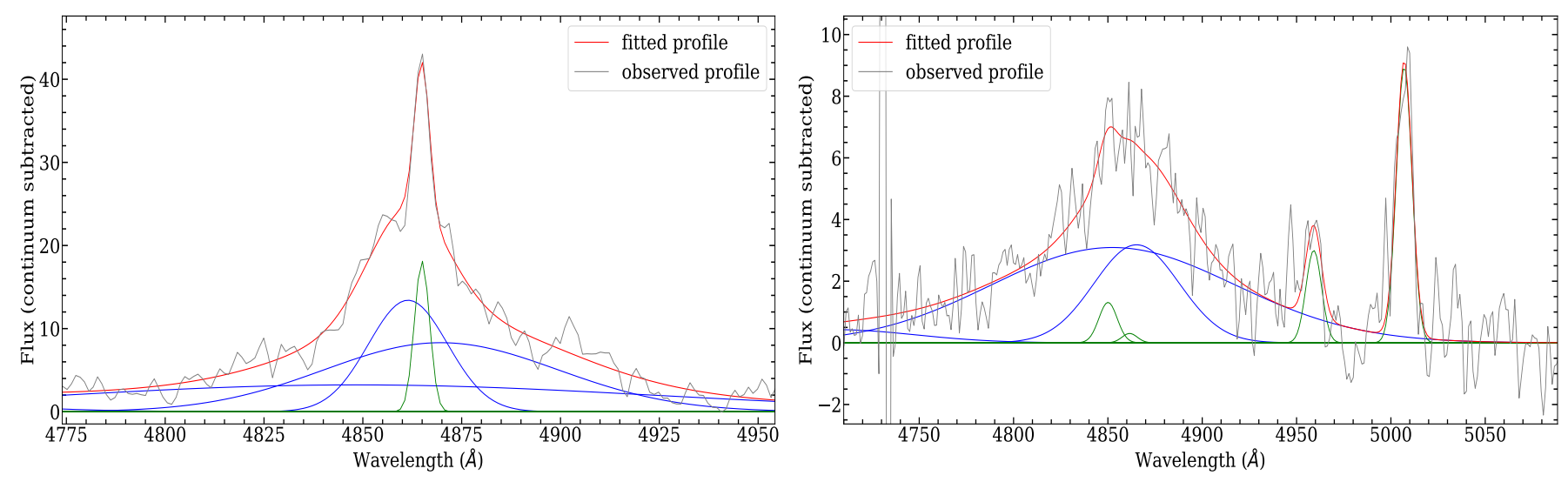

Figure 4. Example of blue (left) and red (right) asymmetric $H \beta$ profiles with AI values -0.14 and +0.12 respectively

The NlSy1 galaxies have been known to have higher accretion rates as compared to the BlSy1, which makes it one of the defining parameters in the classification. These AGN are understood to be younger in age but accreting very fast. In the eigenvector formalism of Boroson \& Green (1992), the Eddington ratio, $R_{E d d}=\frac{L_{B O L}}{L_{E d d}}$ is understood to drive the variations in the parameters. The Eddington ratio is also interpreted as the age of the AGN in some recent works (Grupe, 2004). We estimated the Eddington ratio using optical spectra itself. In Ojha et al. (2020b), $R_{E d d}$ was calculated using X-ray observations which was consistent with the estimation from that obtained from optical parameters hence we did not attempt to estimate $R_{E d d}$ using other methods.

The asymmetry index has been used to trace the signatures of inflowing or outflowing gas in the broad line region of AGN. While the outflow asymmetry in the CIV line, known as the blueshifting is well known and documented (Gaskell \& Goosmann, 2013) and possibly explained by the so called 2 component BLR model, the cause of similar asymmetry in a low ionisation line like $H \beta$ has not been known very well in the literature, although asymmetric $H \beta$ profiles have been known to exist. The characterisation of the AGN in terms of their emission line shapes and shifts and their correlation with physical parameters such as accretion rate etc. has been attempted in (Zamfir et al., 2010) where they conclude that the AGN with $H \beta$ FWHM $\geq 4000 \mathrm{~km} / \mathrm{sec}$ show different characteristics than the ones with lower value of FWHM. We estimated the asymmetry indices for all the AGN in our sample. It has been calculated with different flux values in the recent past (Brotherton, 1996, Marziani et al., 1996). We chose a combination of $75 \%$ and $25 \%$ flux values to estimate the asymmetry index based on previous works. Basically the wavelength at which the broad emission line flux values reach the $75 \%$ and $25 \%$ of the flux values is recorded and the asymmetry index (AI) and Kurtosis Index (KI) are calculated. The correlation of AI with various physical parameters is shown in Figure 5. We also obtained the soft X-ray photon index as it is one of the fundamental components in the 4DE1 formalism. The X-ray photon indices were obtained from (Ojha et al., 2020b). The comparison between X-ray photon indices and the BLR asymmetry provides clues to the connection of the corona 

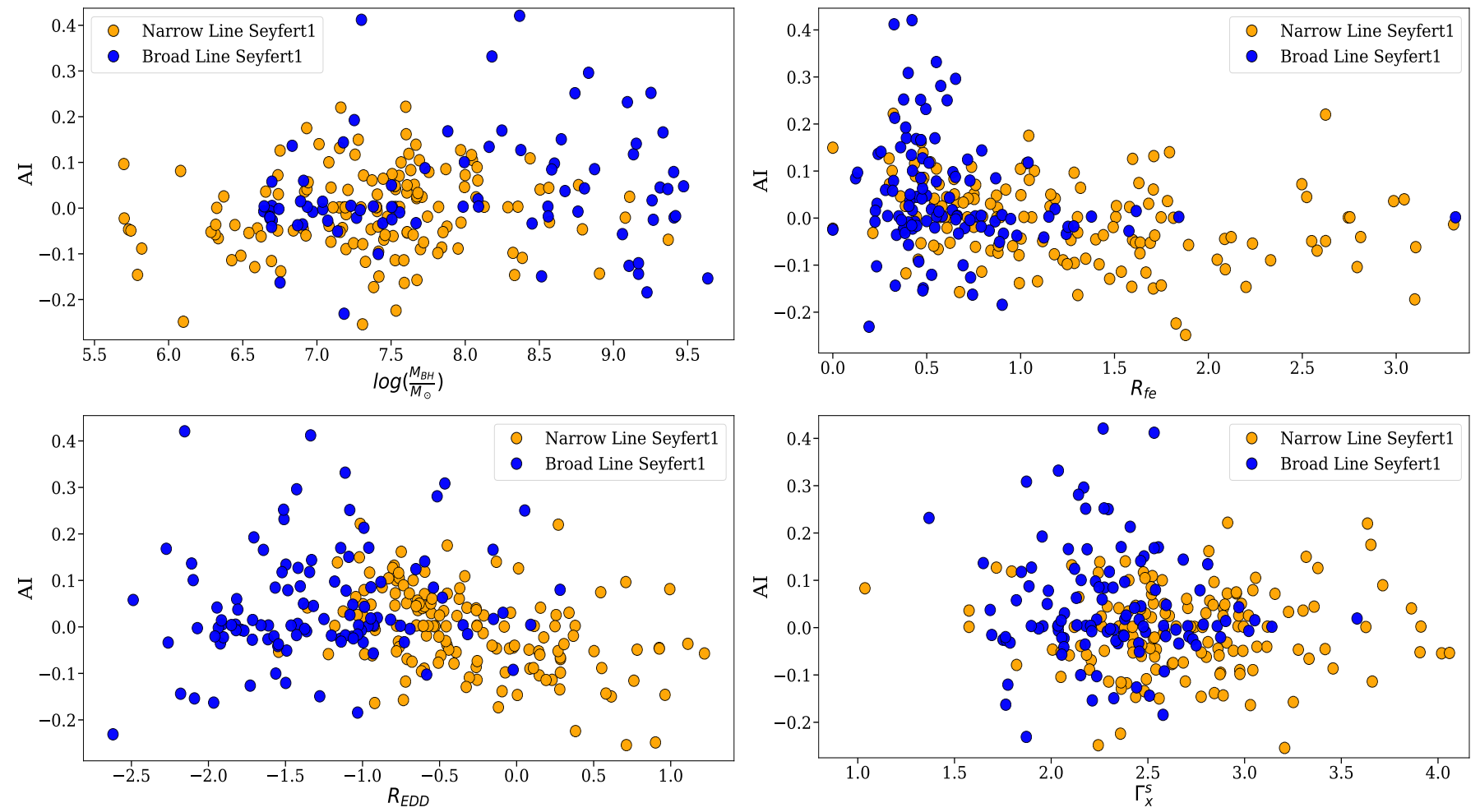

Figure 5. Correlation of asymmetry indices with various parameters for NlSy1 (orange) and BlSy1 (blue) galaxies.

in the accretion disk with the BLR. The distribution of the various parameters for the entire sample of BlSy1 and NLSy1 is presented in Figure 3. To understand the correlation among the various derived parameters, we calculated the spearman rank correlation coefficients. It is a statistical technique used to find out the strength and the direction of the association between two variables.

\section{Results}

The direct correlations point to higher anti correlation of the $H \beta$ FWHM with $R_{f e}$ and $R_{E d d}$ in the NlSy1. While there is a strong anti correlation of FWHM with $R_{f e}$ in both the cases, it is slightly weaker in the case of BlSy1 galaxies. This may be because of the fact that $R_{f e}$ is strongly dependent on the flux of the iron emission line and thus the emission region of NLSy1 being richer in iron content as compared to the BlSy1. The Eddingtion ratio is anti correlated with the $H \beta$ FWHM with the anti correlation coefficient of -0.43 for the whole sample. When the correlation is calculated separately, a positive correlation of +0.33 is present in the case of BlSy1 while it is highly anti correlated with the FWHM of $H \beta$ in the case of NlSy1 galaxies, it's correlation coefficient being -0.84. Most of the NlSy1 galaxies have higher Eddington ratios but lower FWHM than the BlSy1 hence, this skews the results for entire population in the favour of NlSy1 galaxies in this analysis.

More NlSy1 galaxies show blue asymmetries as compared to the BlSy1, which seems quite interesting. Blue asymmetries means there is outflowing gas arising from that region. In the literature (see Panda et al., 2019, Wolf et al., 2020) it has been recently known that the NlSy1 galaxies show more traces of outflow. The FWHM of $\mathrm{H} \beta$ correlates positively, although weakly with the asymmetry index. The correlation coefficent is 0.26 in the case of BlSy1 galaxies while it comes out to be 0.37 in the case of NlSy1 galaxies. Also it is anti correlated with $R_{F e}$ in both the cases of NlSy1 galaxies and BlSy1 galaxies. There has been a postulation to use the $\mathrm{AI}$ in the emission profile as a surrogate parameter in the $4 \mathrm{DE} 1$ formalism. The asymmetry index shows weak correlations and anti correlations with the other known parameters. Thus, we can't conclusively determine the asymmetry index to be a dominating factor in the AGN classification based on this analysis. Soft X-ray photon index has been one of the components of the 4DE1 formalism of Boroson \& Green (1992) hence we tried to correlate 
it with other known parameters for this sample. Surprisingly, there is very weak correlation of the soft X-ray photon index with all the parameters in both the cases.

We tried to see if the differences in physical properties arise even when the NlSy1 galaxies are compared to the general Seyfert galaxies population. Naturally with the NlSy1 galaxies occupying extreme ends in some parameters space, it becomes imperative to understand the properties of these galaxies. In Grupe (2004), and more recently Ojha et al. (2020b) and Waddell \& Gallo (2020) the properties of NlSy1 galaxies have been studied comparatively with BlSy1 galaxies. In Grupe (2004), a sample of $110 \mathrm{X}$-ray selected galaxies was available, which is around 250 in our case, owing to the availability of SDSS sepctrum. The results between the two studies are largely consistent. We conclude from this work that the NlSy1 galaxies are richer in iron content (indicated by their high $R_{f e}$ values), accrete very fast and show more traces of outflow, signified by their blue asymmetries.

\section{Acknowledgments}

The authors acknowledge the financial support provided by DST-SERB (grant no: EMR 2016/001723) for this project.

\section{References}

Bahcall J. N. J., Kozlovsky B.-Z. B.-Z., Salpeter E. E., 1972, ApJ, 171, 467

Bentz M. C., Katz S., 2015, PASP, 127, 67

Bentz M. C., Peterson B. M., Netzer H., Pogge R. W., Vestergaard M., 2009, ApJ, 697, 160

Blandford R. D., McKee C. F., 1982, ApJ, 255, 419

Boroson T. A., Green R. F., 1992, ApJS, 80, 109

Brotherton M. S., 1996, Technical report, THE PROFILES OF Hß AND [O III] X5007 IN RADIOLOUD QUASARS

Du P., Wang J.-M., 2019, ApJ, 886, 42

Gaskell C., 2000, New Astronomy Reviews, 44, 563

Gaskell C. M., Goosmann R. W., 2013, ApJ, 769, 30

Gaskell C. M., Goosmann R. W., 2016, Ap\&SS, 361, 67

Gaur H., Gu M., Ramya S., Guo H., 2019, A\&A, 631, A46

Grupe D., 2004, The Astronomical Journal, 127, 1799

Guo H., Shen Y., Wang S., 2018, PyQSOFit: Python code to fit the spectrum of quasars, Astrophysics Source Code Library (ascl:1809.008)

Kaspi S., Smith P. S., Netzer H., Maoz D., Jannuzi B. T., Giveon U., 2000, ApJ, 533, 631

Kaspi S., Smith P. S., Netzer H., Maoz D., Jannuzi B. T., Giveon U., 2002, ApJ, 533, 631

Marziani P., Sulentic J. W., Dultzin-Hacyan D., Calvani M., Moles M., 1996, ApJS, 104, 37

Marziani P., et al., 2018, Frontiers in Astronomy and Space Sciences, 5, 6

Mathur S., 2000, NewAR, 44, 469

Netzer H., 2015, ARA\&A., 53, 365

Ojha V., Chand H., Krishna G., Mishra S., Chand K., 2020a, MNRAS, 493, 3642 
Ojha V., Chand H., Dewangan G. C., Rakshit S., 2020b, ApJ, 896, 95

Panda S., Marziani P., Czerny B., 2019, ApJ, 882, 79

Rakshit S., Stalin C. S., 2017, ApJ, 842, 96

Rakshit S., Stalin C. S., Chand H., Zhang X.-G., 2017, ApJS, 229, 39

Rakshit S., Stalin C. S., Kotilainen J., 2020, ApJS, 249, 17

Shen Y., et al., 2011, ApJS, 194, 45

Sulentic J. W., 1989, ApJ, 343, 54

Sulentic J. W., Marziani P., 2015, Frontiers in Astronomy and Space Sciences, 2, 6

Sulentic J. W., Zwitter T., Marziani P., Dultzin-Hacyan D., 2000, ApJL, 536, L5

Sulentic J. W., et al., 2017, A\&A, 608, A122

Waddell S. G. H., Gallo L. C., 2020, MNRAS, 498, 5207

Wang J.-M., Du P., Brotherton M. S., Hu C., Songsheng Y.-Y., Li Y.-R., Shi Y., Zhang Z.-X., 2017, Nature Astronomy, 1, 775

Wolf J., et al., 2020, MNRAS, 492, 3580

Yip C. W., et al., 2004, AJ, 128, 585

York D. G., et al., 2000, AJ, 120, 1579

Zamfir S., Sulentic J. W., Marziani P., Dultzin D., 2010, MNRAS, 403, 1759 